\title{
The Shape of Space: Evidence for Spontaneous but Flexible Use of Polar Coordinates in Visuospatial Representations
}

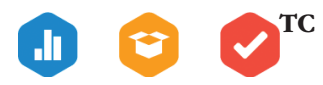

\author{
Sami R. Yousif $i D$ and Frank C. Keil $i$ \\ Department of Psychology, Yale University
}

\begin{abstract}
What is the format of spatial representation? In mathematics, we often conceive of two primary ways of representing 2D space, Cartesian coordinates, which capture horizontal and vertical relations, and polar coordinates, which capture angle and distance relations. Do either of these two coordinate systems play a representational role in the human mind? Six experiments, using a simple visual-matching paradigm, show that (a) representational format is recoverable from the errors that observers make in simple spatial tasks, (b) human-made errors spontaneously favor a polar coordinate system of representation, and (c) observers are capable of using other coordinate systems when acting in highly structured spaces (e.g., grids). We discuss these findings in relation to classic work on dimension independence as well as work on spatial representation at other spatial scales.
\end{abstract}

\section{Keywords}

spatial perception, space, perception, representation, polar coordinates, separability, open data, open materials, preregistered

Received 2/24/20; Revision accepted 9/17/20

A foundational question in the study of any mental process concerns the format of the underlying representation on which that process depends. In our daily use of computers, for example, file formats shape our digital interactions: whether we use a .doc file or a .pdf file affects how we interact with that information, what metadata are stored about that information, and what other processes (i.e., programs) can act on that information. Similarly, the format of mental representations informs where and how those representations are instantiated, whether they are domain general or domain specific, and what kinds of information the mind most naturally represents in the first place. Here, we address this question in the context of spatial representation: Using a simple, novel approach, we revealed the latent format of our most basic visuospatial representations.

Spatial representations are foundational to a diverse array of cognitive processes that are important for aesthetics (Palmer et al., 2013), for representing numbers
(Dehaene et al., 1993; Zorzi et al., 2002), for working memory (e.g., Pertzov \& Husain, 2014), and even for reasoning about social relationships (Parkinson \& Wheatley, 2013). Yet spatial behavior is not always precise: Observers invariably make errors even in straightforward spatial tasks (Hubbard, 2018; McCloskey et al., 1995). For example, in simple spatial-memory tasks, observers tend to remember things as having been closer to the quadrant in which they originated (Huttenlocher et al., 1991; Yousif et al., 2020). And when recalling and perceiving oriented lines, observers make larger errors with diagonal lines compared with horizontal or vertical lines (Appelle, 1972; Li et al., 2003; Olson, 2013). These effects are only a few of many spatial biases, ranging from illusions of 2D area (e.g., Coren \& Girgus, 1978; Yousif \& Keil, 2019), to navigation

Corresponding Author:

Sami R. Yousif, Yale University, Department of Psychology

E-mail: sami.yousif@yale.edu 
errors in 3D environments (Warren et al., 2017; Yousif \& Lourenco, 2017), to misperceptions of an object's center of mass caused by a conflation of $2 \mathrm{D}$ and $3 \mathrm{D}$ forms (Firestone \& Keil, 2016). Many of these errors remain relatively mysterious, although they sometimes speak to the nature of underlying spatial representations (e.g., McCloskey \& Palmer, 1996; Müller \& Wehner, 1998). Here, we exploited these spatial biases: We asked whether such mislocalizations hint at the format of the underlying representation.

\section{Current Study}

In several experiments, observers completed a visualmatching paradigm in which they saw one image (comprising arrangements of shapes or dots within shapes) in a corner of the screen and a corresponding image in the opposite corner of the screen (comprising the same arrangement of shapes but sometimes scaled up or down in size). Observers were then instructed to place a missing shape so that the relative locations of the objects in that image exactly matched the relative locations of the objects in the other image (see Figs. 1 and 2).

The core conclusions of this article rest on an analysis of observers' errors. In short, we measured the correlation between the errors in different dimensions of space (e.g., $x$ vs. $y$ for Cartesian coordinates, angle vs. distance for polar coordinates). If observers represent space via any $2 \mathrm{D}$ coordinate system, and the system is efficient, those two dimensions should be orthogonal. We propose, therefore, that errors in those two dimensions should also be orthogonal (for an example of this kind

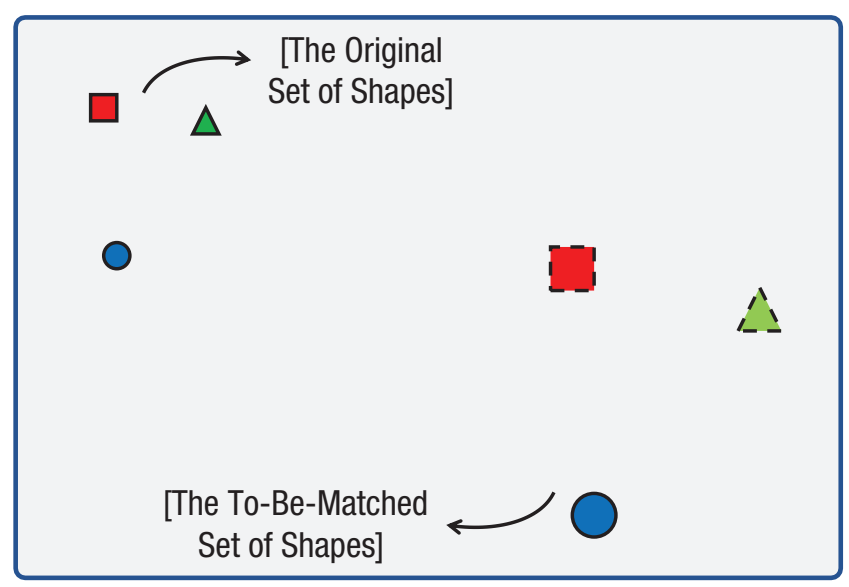

Fig. 1. A schematic of the method. Observers saw a set of shapes (here a triangle, circle, and square) in one corner of the screen and either one or no shapes in the other corner. Their task was to place the missing shapes so that their relative positions matched those in the complete set. In this example, the relative spatial relations of the set in the bottom right are scaled up by a factor of 2 . The dashed lines indicate objects that were not initially present in the display and had to be placed by the observer.

\section{Statement of Relevance}

What is the format of the underlying representation that supports a mental process? This is perhaps the most foundational question that we can ask in any domain - a core goal shared by developmental psychologists, cognitive scientists, and neuroscientists. Just as file formats shape our digital interactions (e.g., whether we use a .doc file or a .pdf file has implications for how we interact with that information, what metadata are stored about that information, and what other processes can act on that information), the format of mental representations is crucial for understanding how complex mental processes are realized in the mind (and how various mental processes interact with one another). This article addresses this fundamental question of format. Here, we provide evidence that visuospatial representations operate spontaneously, but flexibly, in polar space (as opposed to Cartesian space and other plausible spaces).

of analysis, see Bays et al., 2011). In other words, if observers represent space via Cartesian coordinates, we expect that their errors in this coordinate system would be independent, or uncorrelated. Similarly, if observers represent space via Cartesian coordinates, we expect that errors in other coordinate systems (e.g., polar coordinates) would be dependent, or correlated.

We first validated this kind of analysis by running a simulation in specific coordinate systems (see Experiment 0 on our OSF page at https://osf.io/tnhez). Here, we simulated the task-with parameters such as average accuracy set to match that of human participantswith models that operate in either Cartesian or polar coordinates. These simulations demonstrated how the analyses may succeed in principle. Following this, we conducted four experiments showing that human observers spontaneously use polar coordinates (Experiments $1 \mathrm{a}-1 \mathrm{c}$ and $2 \mathrm{a}$ ). We then showed that, despite this default tendency, humans are capable of flexibly operating in other coordinate systems when various levels of spatial structure are imposed on the task environment (Experiments 2a-2c).

\section{Experiments 1a to 1c: Spontaneous Use of Polar Coordinates}

What is the format of human spatial representation? In three experiments, observers completed the same kind of visual-matching task that we simulated in Experiment 0 (see https://osf.io/tnhez). In opposite corners of the screen, there were matched sets of three shapes-a blue 
a

Experiment 1a:

Place Two Shapes

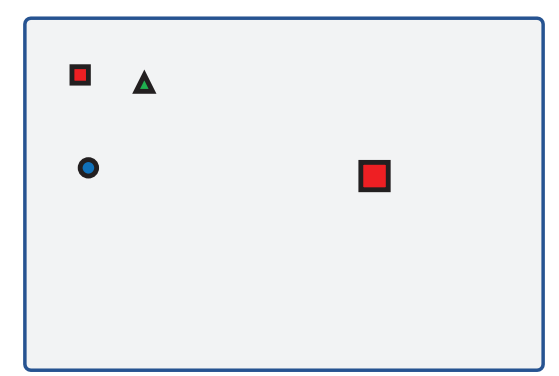

d
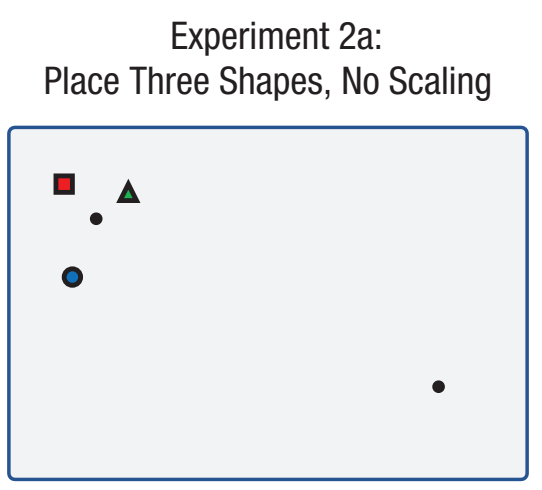

b

Experiment $1 \mathrm{~b}$ : Place Three Shapes

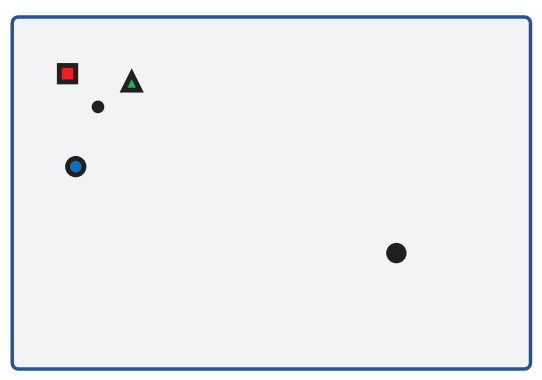

e
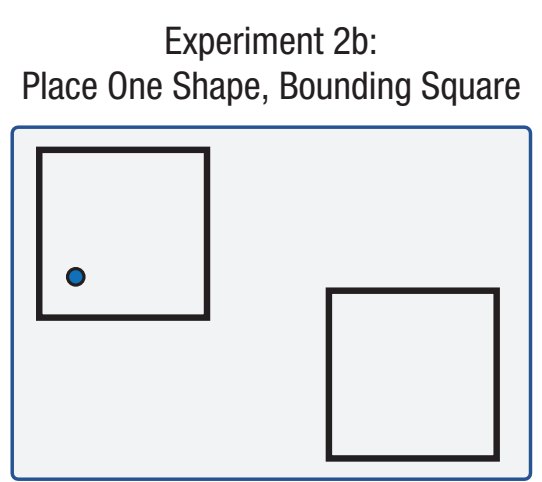

C
Experiment 1c: Place Two Shapes, No Scaling

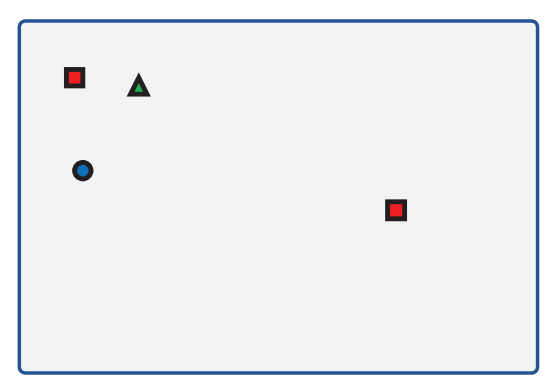

f

Experiment 2c:

Place One Shape, Grid

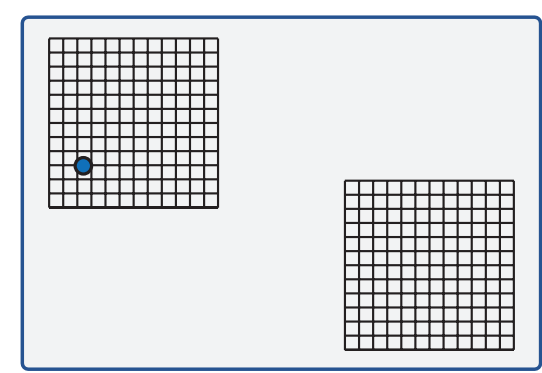

Fig. 2. Schematics of (a) Experiment 1a, (b) Experiment 1b, (c) Experiment 1c, (d) Experiment 2a, (e) Experiment 2b, and (f) Experiment 2c. Items presented here are approximately but not exactly to scale.

circle, a red square, and a green triangle. At first, one of the sets contained either none or one of the three original shapes (depending on the experiment; see below). Observers had to place the missing shapes so that the relative spatial relationships matched those of the set in the opposite corner. Preregistrations for these experiments are available at https://osf.io/tnhez. The experiments were approved by the Yale University Institutional Review Board.

\section{Metbod: Experiment 1a}

observers. Sixteen naive observers from the Yale community completed the experiment in exchange for course credit. This preregistered sample size was chosen before data collection began and was fixed to be identical for each of the in-lab experiments reported here.

Apparatus. The experiment was conducted with custom software written in Python with the PsychoPy libraries (Peirce et al., 2019). Observers sat without restraint approximately $60 \mathrm{~cm}$ from a $43^{\circ} \times 25^{\circ}$ display. All spatial extents reported below are computed on the basis of this distance.

Stimuli. The display on each trial consisted of two sets of shapes; each set contained three unique shapes (a blue circle, a red square, and a green triangle; each with a thin black border) on a gray background $(50 \%$ white, $50 \%$ black). The two sets appeared in opposite corners of the display (counterbalanced so that both sets appeared in each corner an equal number of times). The center of each set of shapes was set to be $5.60^{\circ}$ horizontally and vertically from the center of the display. The position of each shape within the set was randomly determined so that, for the smaller set, a point could appear within $2.24^{\circ}$ horizontally and $2.24^{\circ}$ degrees vertically of that set's center. For the larger set, the locations were matched so that they were exactly twice the distance from their respective center (meaning that points could appear anywhere within $4.48^{\circ}$ horizontally and vertically of the set's center). Random generation of locations was constrained so that no two shapes could appear within $1.25^{\circ}$ of one another (from one object's center to 
another) for the smaller set and double that distance for the larger set. The smaller shapes were set to have a radius of $.36^{\circ}$, and the larger shapes were set to have a radius of $.72^{\circ}$ (here, radius means the distance from the center of the shape to the point along its edge farthest from that center). A different set of randomly generated locations was used for each observer. One set of shapes (the smaller set on half of trials and the larger set on the other half, presented in separate blocks that were counterbalanced across observers as described below) was initially missing two of the three shapes (counterbalanced across trials so that each shape was missing an equal number of times in each block). No other information was visible on the screen at any point. A representative trial can be seen in Figure 1 (see also Fig. 2a).

Procedure. On each trial, observers simply had to place the missing shapes to match the relative location of their corresponding shapes by moving and then clicking the mouse. The missing shape appeared after a mouse click, at which point observers could click additional times or drag and drop the dot to change its location. When observers were satisfied with the missing object's location, they pressed a key to submit their response. If a response was recorded, then the display was replaced with a blank screen for a randomly chosen interval of 0.5 $\mathrm{s}$ to $1.5 \mathrm{~s}$, after which the next trial began. If no response was recorded within $14 \mathrm{~s}$, then a warning to respond more quickly appeared for $5 \mathrm{~s}$ before the next trial began, and that trial was randomly shuffled back into the trial sequence. When that trial was reached, it would use the same set positions (i.e., the quadrants where the sets were located), but a different set of random locations would be generated for the objects themselves (i.e., the shapes would appear in different locations relative to one another).

Design. Each observer completed 192 trials, divided into two equal blocks: 96 small-to-large trials (i.e., with the initially missing object in the larger set) and 96 largeto-small trials (i.e., with the initially missing object in the smaller set). Between the two blocks, a message appeared encouraging observers to rest briefly before continuing. Observers completed four representative practice trials (the data from which were not recorded) before beginning the task.

\section{Results: Experiment 1a}

To assess representational format, we first calculated the absolute error (i.e., ignoring the direction of the error) for each observer and each trial in each of the four relevant dimensions ( $x, y$, angle, and radial distance) relative to where the point should have been.
For example, if the original point was at $[2,2]$, but the second set of shapes was scaled up in size by a factor of 2 , we would calculate error relative to the point [4, 4]. (This is an example of scaling in Cartesian coordinates, but the same logic would apply to polar coordinates.) We then correlated the dimensions of each coordinate system with each other. We then averaged those correlations across individuals and asked whether that average was significantly different from zero.

The coordinate system was always imputed relative to the initially present object. For these analyses, therefore, we calculated a correlation for each person for each point (the first one placed vs. the second one placed) and then averaged those correlations before asking whether they significantly differ from zero. Note, however, that all of the results below replicate if we analyze only the first point observers placed or only the second point.

The results from this experiment can be seen in Figure 3 and Table 1. As shown in the table, Cartesian errors were reliably correlated (mean $r=.19,95 \%$ confidence interval, or $\mathrm{CI}=[.12, .27]), t(15)=5.68, p<.001$, $d=1.42,95 \%$ CI for $d=[0.71,2.11]$, and polar errors were reliably uncorrelated (mean $r=.02,95 \% \mathrm{CI}=$ $[-.02, .05]), t(15)=1.11, p=.28, d=0.28,95 \%$ CI for $d=[-0.23,0.77]$. The difference between these two values was also significant, $t(15)=6.47, p<.001, d=$ $1.62,95 \%$ CI for $d=[0.85,2.36]$. We can also analyze whether noncanonical dimensions are correlated as we would expect them to be. And, indeed, for all of the noncanonical coordinate systems we tested, there was a positive correlation $(p<.001$; see Table 1$)$.

Four factors strengthen the meaningfulness of this null result. First, we always pair a predicted null result in one dimension with a predicted positive result in another; in other words, we are more confident that a null result for polar coordinates is meaningful because Cartesian coordinates yield a positive result. Second, we demonstrated via simulation (in Experiment 0; see https://osf.io/tnhez) that this analysis functions correctly under known conditions. Although we cannot perfectly simulate human behavior, these simulations were conducted in a way that mimicked human behavior (e.g., by matching average error along multiple dimensions). Third, we can ask about other predictions that this view must make. For example, if polar coordinates are implemented in the human mind, then we should expect that any coordinate system not in use should have correlated errors. Whereas the Cartesian system makes for an obvious comparison, there are an infinite number of noncanonical dimensions that we can assess. Most straightforwardly, errors in the angle and $x$ dimensions should be correlated with one another, as well as radial distance and $y$ dimensions, 
a

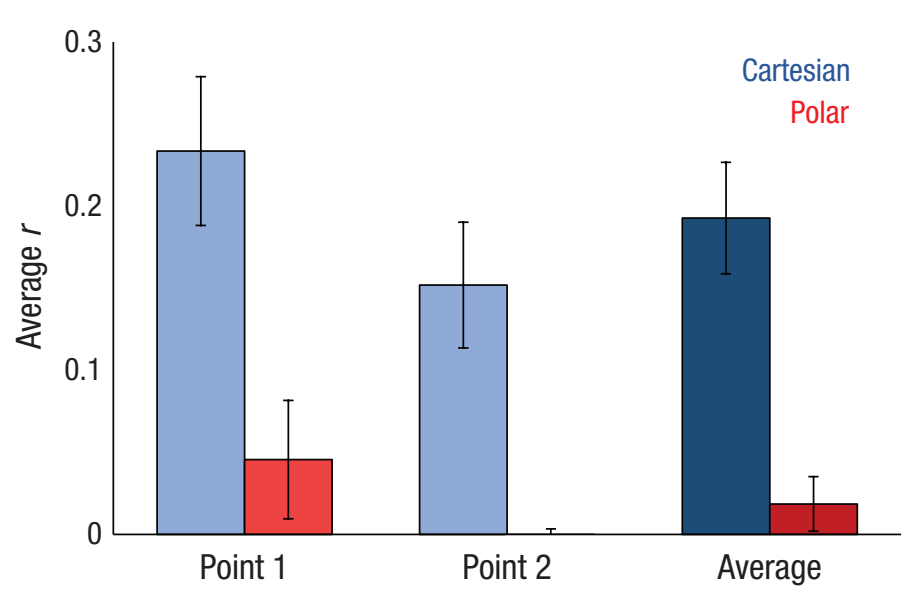

b

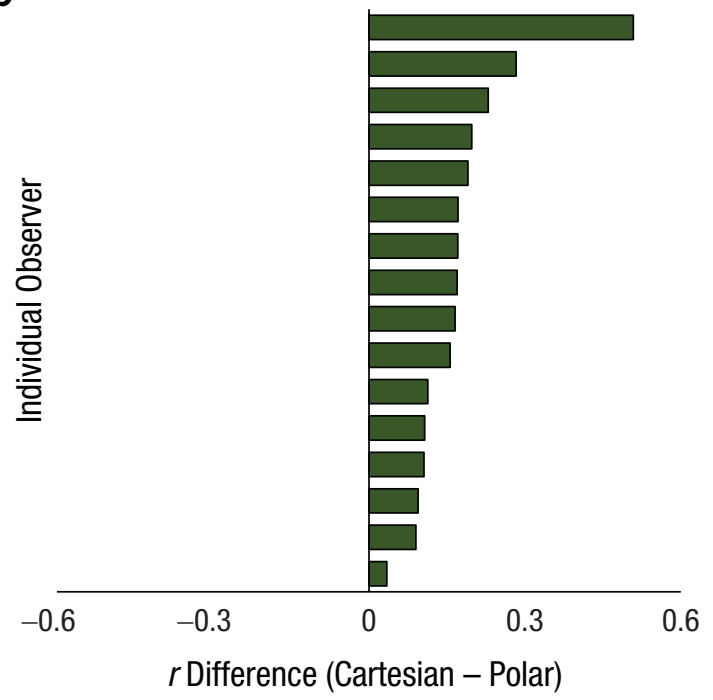

Fig. 3. Results from Experiment 1a. The average correlation for Cartesian and polar errors (a) is broken down by point. (The lighter bars correspond to individual points; the darker bars correspond to the average of those values.) Cartesian correlations are depicted in blue; polar correlations are depicted in red. Error bars represent $\pm 1 S E$. The difference in correlation (b) is shown for each observer. Bars to the right of the $y$-axis indicate a greater correlation for Cartesian dimensions than polar dimensions. For additional information and statistics about these correlation values, see Table 1.

and so on. These correlations are presented along with the other relevant correlations in Table 1; as can be seen, these noncanonical dimensions are correlated as one would expect $(p<.05)$. (As with the analyses above, these $p$ values were derived from a one-sample $t$ test conducted on the correlation values for each observer; we were asking whether, on average, these correlations differed from zero.)

Table 1. Correlations and Significance Values From Experiments 1a to 1c

\begin{tabular}{|c|c|c|c|c|c|c|}
\hline \multirow[b]{2}{*}{ Dimension } & \multicolumn{2}{|c|}{ Experiment 1a } & \multicolumn{2}{|c|}{ Experiment $1 \mathrm{~b}$} & \multicolumn{2}{|c|}{ Experiment 1c } \\
\hline & $r$ & $p$ & $r$ & $p$ & $r$ & $p$ \\
\hline \multicolumn{7}{|c|}{ Primary dimensions } \\
\hline Cartesian & .19 & $<.001$ & .12 & $<.001$ & .20 & $<.001$ \\
\hline Polar & .02 & .283 & -.00 & .710 & .08 & .006 \\
\hline Difference & .17 & $<.001$ & .12 & $<.001$ & .12 & $<.001$ \\
\hline \multicolumn{7}{|c|}{ Other dimensions } \\
\hline$x /$ angle & .30 & $<.001$ & .20 & $<.001$ & .51 & $<.001$ \\
\hline$x /$ distance & .67 & $<.001$ & .66 & $<.001$ & .56 & $<.001$ \\
\hline$y /$ angle & .29 & $<.001$ & .25 & $<.001$ & .43 & $<.001$ \\
\hline$y /$ distance & .66 & $<.001$ & .66 & $<.001$ & .55 & $<.001$ \\
\hline \multicolumn{7}{|c|}{ Rotated Cartesian dimensions } \\
\hline $5^{\circ}$ & .20 & $<.001$ & .18 & $<.001$ & .19 & $<.001$ \\
\hline $15^{\circ}$ & .21 & $<.001$ & .20 & $<.001$ & .19 & $<.001$ \\
\hline $25^{\circ}$ & .22 & $<.001$ & .22 & $<.001$ & .20 & $<.001$ \\
\hline $35^{\circ}$ & .21 & $<.001$ & .24 & $<.001$ & .22 & $<.001$ \\
\hline $45^{\circ}$ & .21 & $<.001$ & .24 & $<.001$ & .24 & $<.001$ \\
\hline
\end{tabular}

Note: "Primary Dimensions" are the most important. The actual numerical difference in the correlation is shown in the "Difference" row; the $p$ value corresponds to the output of the one-sample $t$ test conducted on those difference scores. "Other Dimensions" are correlations between various existing dimensions. "Rotated Cartesian Dimensions" are unique coordinate spaces that were created for the purposes of these analyses. Here, we took ordinary Cartesian space and rotated it by $5^{\circ}, 15^{\circ}, 25^{\circ}, 35^{\circ}$, or $45^{\circ}$ to create new coordinate systems. The aim here was to demonstrate that the lack of correlation for polar coordinates is special-as all other combinations of dimensions yield positive correlations. 
a

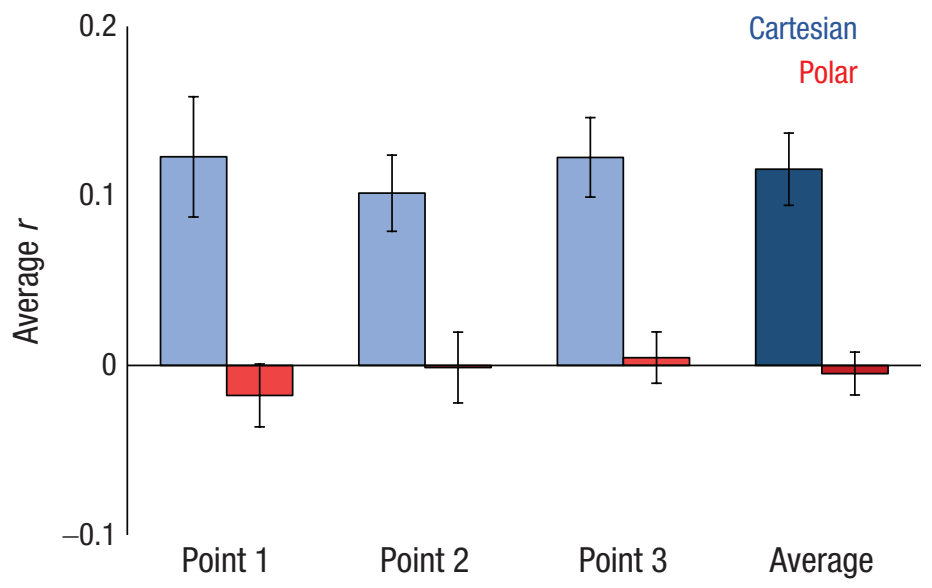

b

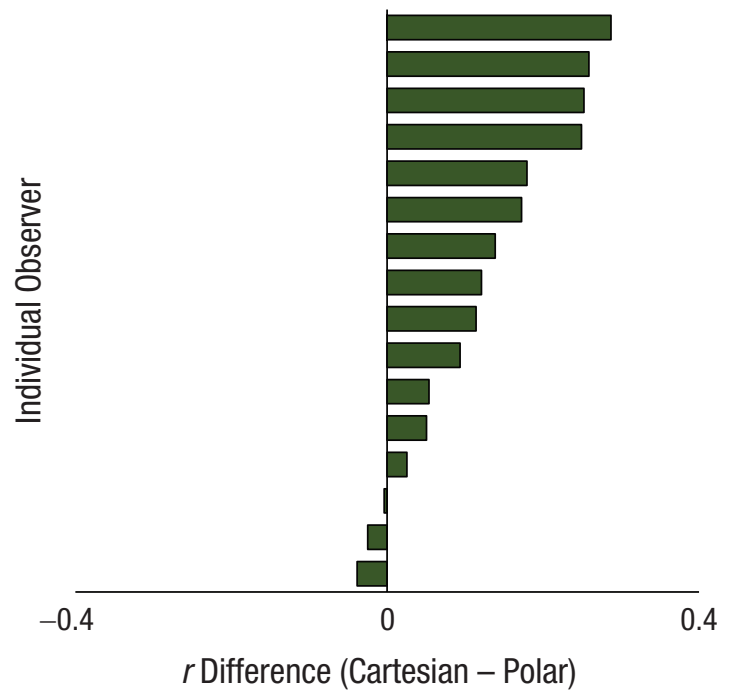

Fig. 4. Results from Experiment 1b. The average correlation for Cartesian and polar errors (a) is broken down by point. (The lighter bars correspond to individual points; the darker bars correspond to the average of those values.) Cartesian correlations are depicted in blue; polar correlations are depicted in red. Error bars represent $\pm 1 S E$. The difference in correlation (b) is shown for each observer. Bars to the right of the $y$-axis indicate a greater correlation for Cartesian dimensions than polar dimensions; the bar to the left indicates the reverse. For additional information and statistics about these correlation values, see Table 1.

Fourth, we can ask about other nonexistent dimensions. For example, it is possible in theory to represent 2D space in a Cartesian-esque format that is rotated, for example, $5^{\circ}$ clockwise, $15^{\circ}$ clockwise, and so on. In other words, we imagined a Cartesian space that was rotated $5^{\circ}$; we then recalculated the coordinates for every error as if they existed in this nonexistent space. Then, we asked whether these nonexistent dimensions properly yield positive correlations-and, indeed, they do $(p<.001)$. The $p$ values of these average correlations are also plotted in Table 1 (as rotated Cartesian dimensions). Therefore, polar representations seem to underlie human errors, as errors in polar coordinates seem to be uniquely uncorrelated-compared not only with Cartesian coordinates but also with other noncanonical 2D spaces.

\section{Metbod: Experiment 16}

This experiment was identical to Experiment 1a, except as noted. Sixteen new observers participated; this preregistered sample size was chosen to match that of Experiment 1a. In this experiment, three shapes (as opposed to two) were initially absent from one of the sets (see Fig. 2b). Observers had to place all three shapes back on each trial. To guide them, we placed a single black dot in the center of the three shapes (for both sets of shapes). Observers did not know which object would appear first when they clicked for the first time (although the objects always appeared in the same order: blue circle, green triangle, red square). After they clicked, they could see the object and then adjust as needed. Observers could press the space bar to lock in the location of the first object, at which point clicking again would cause another shape to appear. They could then adjust the location of that object in the same way. This continued until all three objects were placed and the observer locked in their final response.

\section{Results: Experiment 16}

The analyses for this experiment were identical to the analyses of Experiment 1a, except that there were more points to analyze because observers placed three objects on each trial instead of two. To simplify these analyses, we present the average values for all three points. However, the results are qualitatively identical for each of the three points (as is readily apparent in Fig. 4). The placement error for each object on each trial was always analyzed relative to the central anchor dot.

The results from this experiment can be seen in Figure 4 and Table 1 . As shown in the table, Cartesian errors were reliably correlated (mean $r=.12$, 95\% $\mathrm{CI}=[.07, .16]), t(15)=5.44, p<.001, d=1.36,95 \% \mathrm{CI}$ for $d=[0.66,2.04]$, and polar errors were reliably uncorrelated (mean $r=-.00,95 \% \mathrm{CI}=[-.03, .02]$ ), $t(15)=$ $0.38, p=.71, d=0.10,95 \%$ CI for $d=[-0.59,0.40]$. The difference between these two values was also significant, $t(15)=4.55, p<.001, d=1.14,95 \%$ CI for $d=$ $[0.49,1.76]$. As with the previous experiment, we could 
also analyze whether noncanonical dimensions are correlated as we should expect them to be. And, indeed, all the noncanonical coordinate systems tested were positively correlated $(t \mathrm{~s}>5.40, p s<.001, d s>1.30$; see Table 1). Although this task served mostly as a replication of Experiment 1a, the experience of completing the task was quite different. The presence of only a central anchor point may have altered observers' strategies and suggests that the results of Experiment 1a cannot be explained by appeal to some idiosyncratic task demand (and that, on the contrary, this pattern of results may be far more general).

\section{Method: Experiment 1c}

This experiment was identical to Experiment 1a, except as noted. Sixteen new observers participated; this preregistered sample size was chosen to match that of Experiment 1a. In both prior experiments, one of the sets was scaled to be larger than the other. Such scaling does not affect Cartesian and polar coordinates equally. When spaces are scaled in size, both the dimensions of Cartesian space will change (unless a point lies directly along an axis). However, only one of the dimensions of polar space will change; the angle remains constant. Therefore, these results might occur because polar coordinates are simply a more convenient format for spatial translation. Here, we used an identical task but without the size-translation component: Observers had to match two identical sets of shapes. The two different sets of shapes were not scaled in size; instead, they were spatially identical. We used exactly the same parameters as in Experiment 1a, except that the sizes of both sets were scaled up to be equal to the size of the larger set in Experiment 1a (see Fig. 2c).

\section{Results: Experiment 1c}

The analyses for this experiment were identical to the analyses of Experiment 1a. Again, to simplify these analyses, we present the average values for the two placed points. However, the results are qualitatively identical for each of the points (as is readily apparent in Fig. 5).

The results from this experiment can be seen in Figure 5 and Table 1. As can be seen in the table, Cartesian errors were reliably correlated (mean $r=.20$, 95\% CI $=$ $[.15, .25]), t(15)=8.63, p<.001, d=2.16,95 \% \mathrm{CI}$ for $d=$ $[1.24,3.06]$, and polar errors exhibited small correlations (mean $r=.08,95 \% \mathrm{CI}=[.03, .13]$ ), $t(15)=3.22, p=.006$, $d=0.81,95 \%$ CI for $d=[0.23,1.36]$. The difference between these two values was significant, $t(15)=7.40$, $p<.001, d=1.85,95 \%$ CI for $d=[1.02,2.66]$. As with the previous experiment, we could also analyze whether noncanonical dimensions are correlated as we should expect them to be. And, indeed, for all the noncanonical coordinate systems we tested, there was a positive correlation ( $t$ s $>4.40, p s<.001, d s>1.05$; see Table 1 ). The observed correlation for polar errors was driven largely by a single observer who was an outlier in terms of overall accuracy. However, we did not preregister any exclusion criteria for accuracy for our in-lab experiments. Despite this anomaly, we note that each of the observers still exhibited a higher Cartesian correlation than polar correlation. Therefore, these results once again reveal evidence of spontaneous use of polar coordinates to represent visual space. Here, crucially, we demonstrate small correlations in polar dimensions even when observers completed no size-translation task at all. This suggests that the prior results are not explained by an advantage of one coordinate system during spatial translation; however, the slight polar correlations observed here may suggest that the translations do impact behavior. This possibility was further explored in Experiment 2a.

\section{Discussion: Experiments 1a to 1c}

Experiments 1a to $1 \mathrm{c}$ demonstrate that even small errors made by observers in a maximally simple task contain a wealth of information; indeed, these errors may reveal the canonical format of the spatial representations. Experiments $1 \mathrm{a}$ and $1 \mathrm{~b}$ demonstrate that observers use polar coordinates when scaling spaces up or down in size, whether they are placing them relative to one another or to a single, central landmark. Experiment 1c demonstrates that observers may use polar coordinates even when matching two spatially identical displays. Together, these three experiments provide evidence that observers spontaneously use polar coordinates to represent visual space.

\section{Experiments 2a to 2c: Flexibility of Representation}

In tasks with minimal intervening spatial structure (Experiments 1a-1c), observers automatically operate in polar coordinates. But how flexibly do people engage different coordinate systems across different layouts and reference frames? People's use of polar coordinates might be highly inflexible; that is, regardless of the surrounding spatial environment, people will use only polar coordinates. Or people might spontaneously use polar coordinates as a default representation but may flexibly represent space in other coordinate systems if the surrounding spatial environment strongly suggests 
a

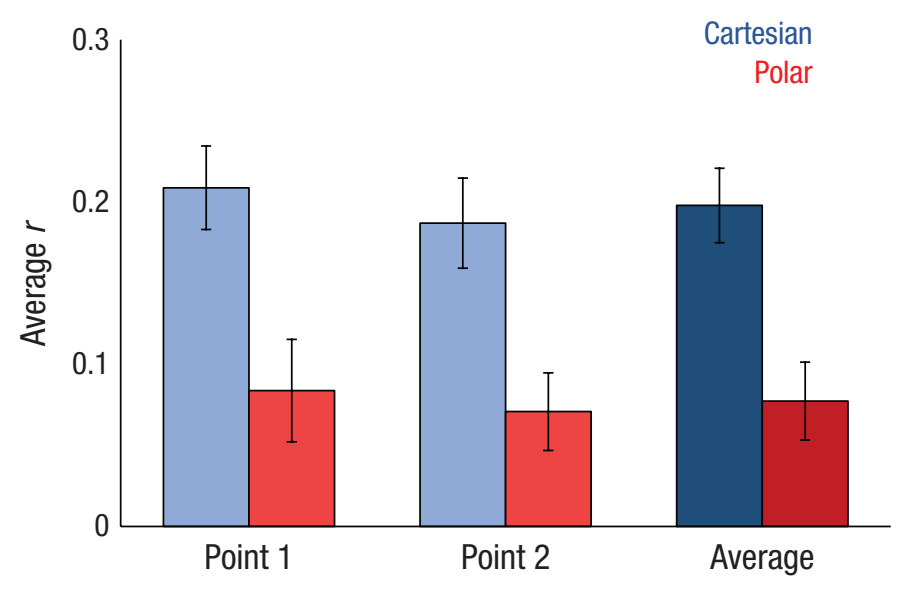

b

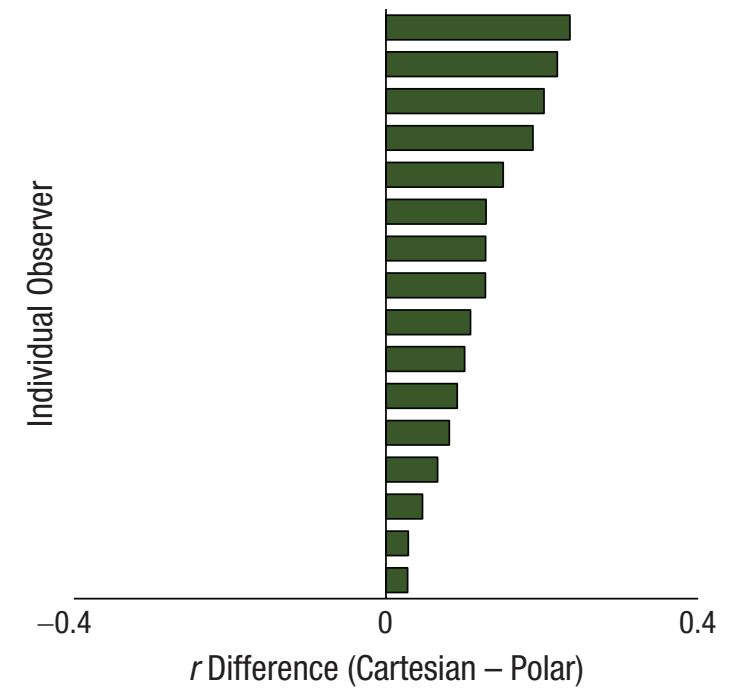

Fig. 5. Results from Experiment 1c. The average correlation for Cartesian and polar errors (a) is broken down by point. (The lighter bars correspond to individual points; the darker bars correspond to the average of those values.) Cartesian correlations are depicted in blue; polar correlations are depicted in red. Error bars represent $\pm 1 S E$. The difference in correlation (b) is shown for each observer. Bars to the right of the $y$-axis indicate a greater correlation for Cartesian dimensions than polar dimensions; the bar to the left indicates the reverse. For additional information and statistics about these correlation values, see Table 1.

a particular system. Here, we used the same spatial matching task as before but in environments with varying degrees of spatial structure. In Experiment 2a, we replicated the findings of Experiment 1; in Experiment $2 \mathrm{~b}$, we imposed moderate structure in the form of a bounding square; and in Experiment 2c, we imposed strong structure in the form of a grid. Preregistrations for these experiments are available at https://osf.io/ tnhez. The experiments were approved by the Yale University Institutional Review Board.

\section{Metbod: Experiment 2a-online replication, minimal structure}

This experiment was identical to Experiment 1c, except as noted. Fifty new observers participated. Of the original sample of 50 , three observers were excluded for failing to complete the task, and a further four observers were excluded for being outliers for overall accuracy; this resulted in a final sample of 43 observers. Unlike the previous experiments, this experiment was conducted online via Amazon Mechanical Turk. (This is because these data were collected in response to a revision request that we received around the onset of the COVID-19 pandemic. Because we were no longer able to collect data in the lab, we opted to convert our experiments to operate online. This is also why we took care to first replicate our original findings on this new platform before attempting to extend them further.) This experiment was run using custom software written in JavaScript.

As much as possible, we tried to match the design of the original experiments online. However, because of uncertainty about the viewing conditions of our observers (given differences in Web browsers, etc.), we cannot know for sure the exact stimulus dimensions and so on. Here, observers placed three points relative to a single, central anchor point (procedurally identical to Experiment 1b; see Fig. 2d). The only other substantive change we made to the task was that we had observers complete only 48 trials compared with the original 192. Note that even though we collected fewer trials per observer, our sample size was also many times larger. Observers had $20 \mathrm{~s}$ to make a response before that trial was skipped and replaced; data from these missed trials were discarded. (The preregistration for this experiment states that observers would have $7 \mathrm{~s}$ to respond before a trial was skipped. However, this was an error. This should have said that observers were given roughly $7 \mathrm{~s}$ per shape they had to place to be consistent with all the other experiments. Because they were placing three shapes, they had $20 \mathrm{~s}$, rounded down from 21.) To account for increased noise in online data collection, we added exclusion criteria at both the trial level and subject level. Any trial with an overall accuracy greater than 2.5 standard deviations from that observer's mean was discarded; any observer 
with an overall accuracy greater than 2.5 standard deviations away from the group mean was discarded.

\section{Results: Experiment 2a-online replication, minimal structure}

The results from this experiment can be seen in Figure 6 . The analyses for this experiment were identical to the analyses for Experiment 1b. Again, to simplify these analyses, we present the average values for all three points. However, the results are qualitatively identical for each of the three points. As can be seen in the figure, Cartesian errors were reliably correlated (mean $r=$ $.13,95 \% \mathrm{CI}=[.09, .18]), t(42)=6.69, p<.001, d=1.02$, $95 \%$ CI for $d=[0.65,1.39]$, and polar errors were reliably uncorrelated (mean $r=.02,95 \% \mathrm{CI}=[-.02, .06]$ ), $t(42)=1.03, p=.31, d=0.16,95 \%$ CI for $d=[-0.15$, $0.46]$. The difference between these two values was also significant, $t(42)=6.03, p<.001, d=0.92,95 \%$ CI for $d=[0.56,1.27]$. These results replicate the findings of Experiment 1, suggesting that the pattern of results that we observed in prior experiments generalizes across testing environments. Further, these results show that the lack of polar correlation was not dependent on the size-translation task.

\section{Metbod: Experiment 2b-bounding square, moderate structure}

This experiment was identical to Experiment 2a, except as noted. Fifty new observers participated; this preregistered sample size was chosen to be identical to that of Experiment 2a. Of the original sample of 50, one observer was excluded for failing to complete the task, and a further six observers were excluded for being outliers for overall accuracy; this resulted in a final sample of 43 observers.

Whereas in the previous experiment, observers placed three points relative to a central anchor point, observers in this experiment matched the location of only one point within a square frame (see Fig. 2e). The goal of this experiment was to provide observers with a moderate level of spatial structure. Because observers were placing only a single shape (as opposed to three shapes), they had only $7 \mathrm{~s}$ to respond before a trial was skipped. Analyses in this experiment had to be conducted relative to the center of the square.

\section{Results: Experiment 2b-bounding square, moderate structure}

The results from this experiment can be seen in Figure $6 \mathrm{~b}$. The analyses for this experiment were identical to the analyses for Experiment 2a, except that observers placed only a single point on each trial. As can be seen in the figure, we observed for the first time a case in which Cartesian errors were uncorrelated (mean $r=$ $.04,95 \% \mathrm{CI}=[-.03, .11]), t(42)=1.24, p=.22, d=0.19$, $95 \%$ CI for $d=[-0.11,0.49]$, and polar errors were correlated (mean $r=.16,95 \% \mathrm{CI}=[.10, .21]), t(42)=6.23$, $p<.001, d=0.95,95 \%$ CI for $d=[0.59,1.31]$. The difference between these two values was significant, $t(42)=2.98, p=.005, d=0.46,95 \%$ CI for $d=[0.14$, 0.77]. This reversal is significant for two reasons: (a) It validates the analysis in the first place, demonstrating that this way of analyzing errors can reveal different strategies that observers may take, and (b) it suggests that whereas observers may spontaneously use polar coordinates in environments with minimal spatial structure, they are capable of flexibly using different spatial representations when the environment strongly implies such representations.

\section{Method: Experiment 2c-grid, maximal structure}

This experiment was identical to Experiment 2b, except as noted. Fifty new observers participated; this preregistered sample size was chosen to be identical to that of Experiment 2a. One observer was excluded for failing to complete the task, and a further three observers were excluded for being outliers for overall accuracy; this resulted in a final sample of 46 observers.

Whereas in the previous experiment, observers placed one point within a bounding square, observers here placed one point on top of a grid (see Fig. 2f). The goal here was to provide observers with a high level of spatial structure (meaning that, unlike in the prior experiments, observers had enough spatial information to make very exact estimates of the object's position-this is why we imposed a time limit on responses; although observers could in theory respond with almost perfect accuracy, this imposed time limit was meant to force small errors).

\section{Results: Experiment 2c-grid, maximal structure}

The results from this experiment can be seen in Figure 6c. The analyses for this experiment were identical to the analyses for Experiment $2 \mathrm{~b}$. As can be seen from the figure, we once again observed that Cartesian errors were uncorrelated (mean $r=.00,95 \% \mathrm{CI}=[-.05, .06]$ ), $t(45)=0.14, p=.89, d=0.02,95 \%$ CI for $d=[-0.27$, 0.31 , and polar errors were correlated (mean $r=.32$, $95 \% \mathrm{CI}=[.27, .37]), t(45)=12.84, p<.001, d=1.89$, 
a

\section{Experiment 2a:}

Minimal Structure
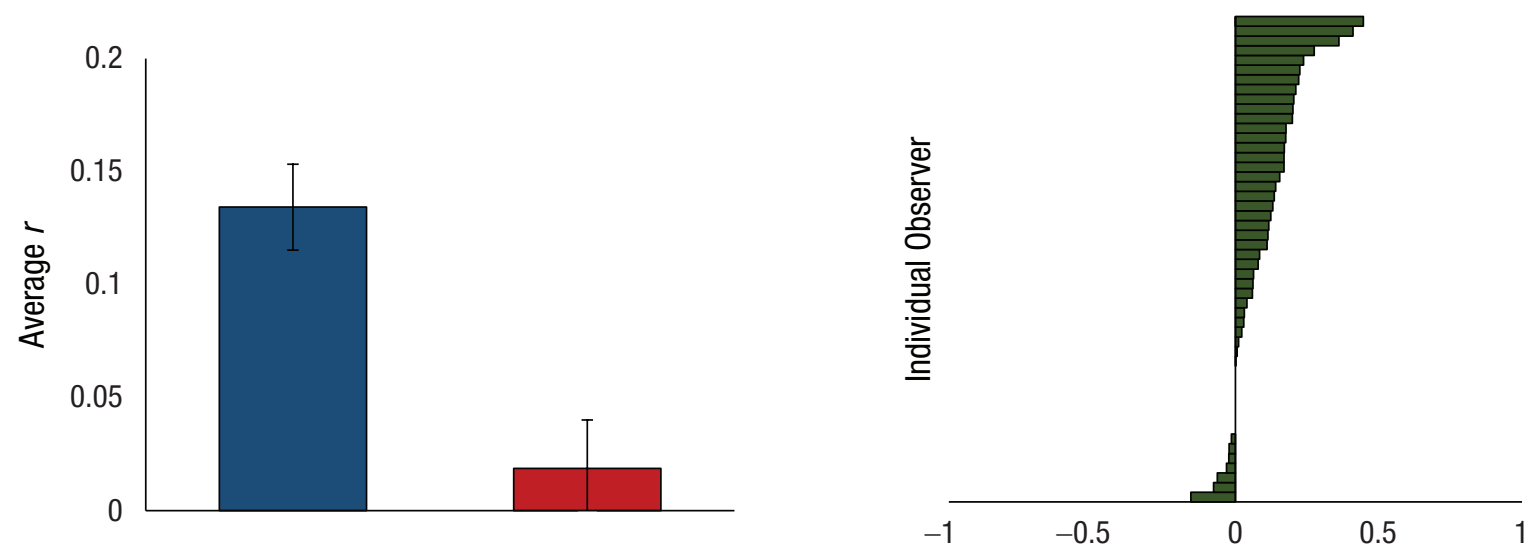

b

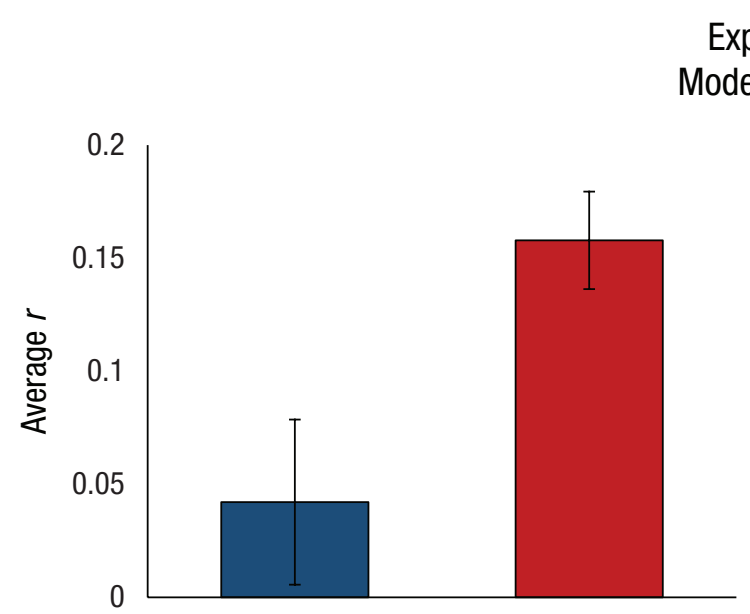

Experiment 2b:

Moderate Structure

C

Experiment 2c:

Maximal Structure
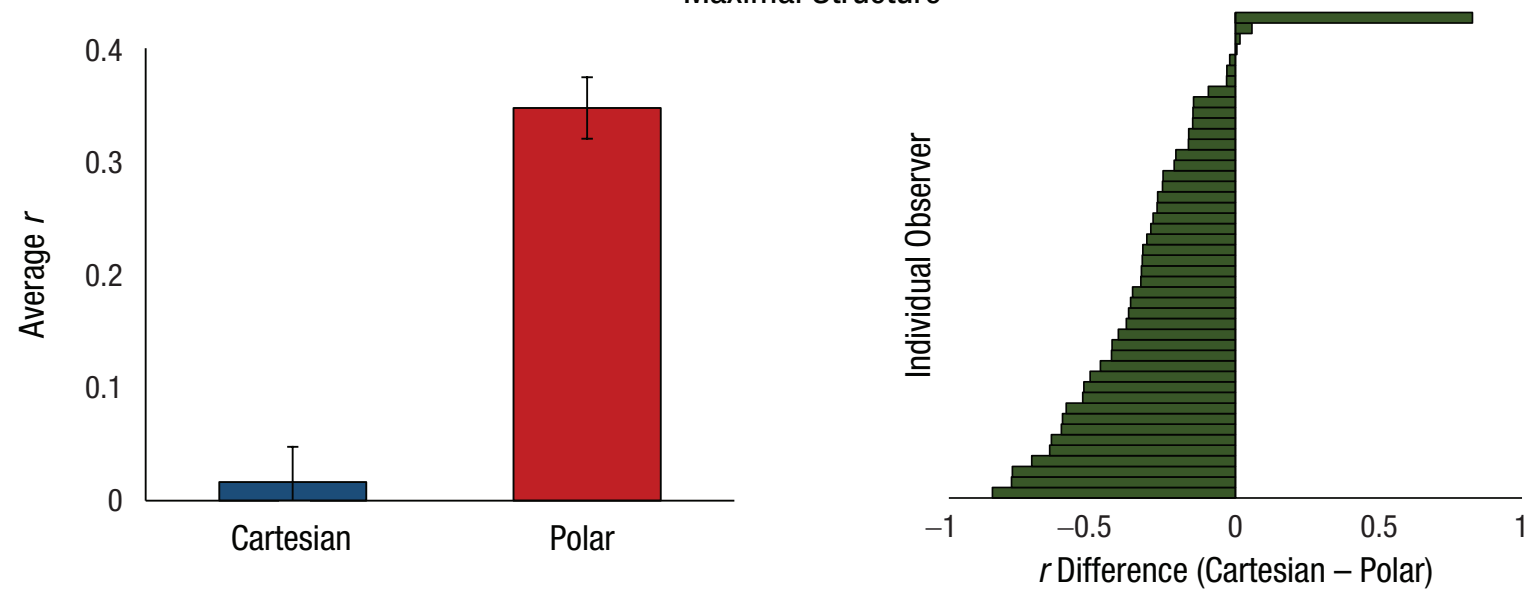

Fig. 6. Results from (a) Experiment 2a, (b) Experiment 2b, and (c) Experiment 2c. The average Cartesian correlations and average polar correlations are shown on the left-hand side. Error bars represent $\pm 1 S E$. Differences in correlations for each observer are shown on the right-hand side. Bars to the right of the $y$-axis indicate a greater correlation for Cartesian dimensions than polar dimensions; bars to the left indicate the reverse. 
95\% CI for $d=[1.40,2.37]$. The difference between these two values was significant, $t(45)=7.61, p<.001, d=$ $1.12,95 \% \mathrm{CI}$ for $d=[0.75,1.49]$. Once again, these results demonstrate that spatial structure can meaningfully impact observers' representations of space.

\section{Discussion: Experiments 2a to $2 c$}

In a series of three experiments, we showed how varying levels of spatial structure influence the kind of coordinate systems that observers use to localize objects. In Experiment 2a, we replicated the findings of Experiments $1 \mathrm{a}$ to $1 \mathrm{c}$, demonstrating that in the absence of strong spatial cues, observers will spontaneously use polar coordinates. But in Experiments $2 \mathrm{~b}$ and $2 \mathrm{c}$ with increasing levels of spatial structure-and, in particular, structure that may lend itself to Cartesianesque representations-observers' patterns of errors revealed an increasing shift toward Cartesian coordinates. These results validate the previous analyses while revealing the boundary conditions of the use of polar coordinates.

Another way of thinking about the results of Experiments $2 \mathrm{~b}$ and $2 \mathrm{c}$ is with respect to reference frames (e.g., Farah et al., 1990). In Experiments 1a to 1c and $2 \mathrm{a}$, the only possible referents (or reference frame) that observers can use to situate the placement of new objects are single points in space (i.e., the already visible objects). In Experiments $2 \mathrm{~b}$ and $2 \mathrm{c}$, by contrast, observers have an entire bounded region (i.e., the square/grid) with which to situate the new object. Note here that our work does not imply that observers do or should use only one coordinate system or one reference frame. Quite the opposite; our approach is meant to be flexible: In principle, our analyses can be conducted relative to any point in space and with respect to any reference frame. And, in practice, this is clearly the case: The fact that we observed a qualitatively different pattern of results in Experiments $2 \mathrm{~b}$ and 2c suggests that observers are clearly capable of representing space within different frames of reference.

\section{General Discussion}

We first demonstrated that analyses of errors have the potential to reveal a representational format when that representational format is known (Experiment 0; see https://osf.io/tnhez). We then applied this insight to six experiments with humans. In Experiments $1 \mathrm{a}$ and $1 \mathrm{~b}$, we found converging evidence of polar coordinates in a simple visual-matching paradigm in which no representational format was implied. In Experiment 1c, we showed that these prior results cannot be explained by the size-translation task itself. In the following three experiments (Experiments 2a-2c), we explored whether observers flexibly use different coordinate systems depending on the spatial context. With high levels of spatial structure (i.e., imposing the spatial-matching task on a grid), observers' pattern of errors suggested the use of Cartesian rather than polar coordinates. Collectively, these results demonstrate spontaneous, but flexible, use of polar coordinates.

These results are far from obvious: All dimensions could have been consistently correlated; or none could have been; or contrary to what we found, Cartesian coordinates could have been uniquely uncorrelated. Yet the same pattern held across many variations of experiments (namely, Experiments $1 \mathrm{a}-1 \mathrm{c}$ and $2 \mathrm{a}$ ), suggesting a robust set of findings. That said, any one of these results in isolation should be interpreted cautiously. We are comfortable interpreting the lack of correlation for polar dimensions as speaking to representational format only because (a) this result was highly replicable across many observers and several unique experiments, (b) we were able to conduct simulations indicating that this analysis could work in principle, (c) our analyses in Experiments 2a through 2c revealed a change in representation in practice, and (d) we assessed a number of other dimensions that also could have been uncorrelated (yet never were).

Nevertheless, the present work depended on a single paradigm. Although this paradigm is revealing, future work may still fruitfully seek converging evidence to support this view. For example, we now know that observers are capable of flexibly swapping between coordinate systems depending on the context. Yet further investigation may be able to address what specific context information may be sufficient to induce a change in representational format as well as the interface between small-scale and large-scale representations (e.g., could we use this analysis to measure representational format in navigable environments?). We see the present work as a first step-one that opens the door to many other lines of inquiry.

\section{Relation to prior work}

These findings relate to prior studies on spatial localization and mislocalization (Huttenlocher et al., 1991; Langlois et al., 2017; Wedell et al., 2007; Yousif et al., 2020), some of which specifically address polar coordinates as a candidate for visuospatial representation (Huttenlocher et al., 1991; Yousif et al., 2020; see also Yang \& Flombaum, 2018). Most notably, Huttenlocher and colleagues (1991) relied on similar correlation analyses to make claims about representational format. However, those results were indecisive for a few reasons. First, the primary aim in their research was to 
understand the origin of spatial biases, not to document the format of visuospatial representations. Second, their conclusions depend solely on a null result, without making predictions about or assessing other dimensions or other spatial contexts (whereas the present work tested many positive predictions and also tested many different spatial contexts). Third, they assessed dimension independence only in circular spaces (whereas we specifically sought to test unbounded spaces), and fourth, they assessed errors in memory, whereas all of our tasks intentionally minimized memory demands.

These results may also bear on spatial representation on larger scales or in 3D environments (e.g., for purposes of navigation; see Moser et al., 2008, 2014). One relevant proposal suggests that we use a network-like cognitive graph for large-scale spatial systems. These graphs especially prioritize angle and distance information between known locations (Ericson \& Warren, 2020; Warren et al., 2017; but see also Gallistel, 1990; Kuipers et al., 2003; O'Keefe \& Nadel, 1978). Of course, this resembles polar coordinates, which are nothing more than angle and distance vectors. ${ }^{1}$ Could the same highly general representational format be employed in both small-scale (i.e., visual) and large-scale (i.e., navigable) environments? Future work may shed light on the continuity of these representations across scales or on the translation of information between small-scale and large-scale layouts (e.g., as when reading maps).

The approach here also relates to classic work on integral versus separable dimensions (Garner \& Felfoldy, 1970; for a review, see Algom \& Fitousi, 2016). Traditionally, researchers investigate integrality and separability in one of two ways: either via Stroop effects or via speeded classification. Dimensions that interfere with one another would be considered integral; dimensions that do not would be considered separable. The error-independence analyses we used here might provide a novel method for assessing integrality versus separability (see also Bays et al., 2011); in principle, all three analyses should yield converging results. That said, these classic paradigms would have been insufficient to address our key questions. Space is not like other dimensions in that any point in space could be simultaneously represented in an infinite number of $2 \mathrm{D}$ spaces. Because our task provides a blank slate with which we can simultaneously analyze all possible dimensions at once, it provides a unique advantage over earlier tasks. Stroop and speeded-classification paradigms, in contrast, require precommitment to the relevant dimensions. Nevertheless, future work may link our approach to the integrality-versus-separability approach.

\section{On format}

Throughout the article, we have focused primarily on a contrast between polar coordinates and Cartesian coordinates (and, briefly, other noncanonical coordinate systems). However, this work also speaks to a possible contrast between the use of some coordinate system and no coordinate system at all. Indeed, it is possible that space could be represented only in coarse spatial terms (e.g., "that point was generally up and to the left"; see Huttenlocher et al., 1991). Both sets of experiments reported here, by contrast, suggest a reliance on a specific coordinate system. Even if participants flexibly rely on multiple coordinate systems, their patterns of errors have still revealed a fundamental regularity: Locations in the mind are represented as variables in a $2 \mathrm{D}$ vector. In some ways, these insights were presaged by the study of patient A. H., who exhibited profound localization deficits that often involved "mirror flipping" points in space (McCloskey \& Palmer, 1996; McCloskey et al., 1995). For example, if instructed to recreate the location of a point offset to the left, A. H. might place a point in the same relative location but offset to the right instead. Such errors suggest that space is being represented in some precise format but one that can be manipulated (akin to flipping the sign of a variable). This work also suggests that space is represented via independent dimensions (or else it would be impossible to make an error in one dimension while acting precisely in another). The present work builds on the study of patient A. H. by offering-for the first time-evidence that the precise coordinate systems underlying visuospatial representations are readily recoverable, even in simple psychophysical tasks.

\section{Conclusion}

We depend on our ability to accurately perceive and represent space; yet, naturally, our percepts and our representations are imprecise. Here, we have shown how errors in the simplest possible spatial tasks contain significant clues to the underlying format of our most primitive visuospatial representations. The present work lays the groundwork for considering domaingeneral mechanisms that may underlie many kinds of spatial biases (e.g., those pertaining to location vs. those pertaining to orientation) across many different spatial scales (e.g., small-scale visual environments vs. large-scale navigable environments). More consequentially, the present work demonstrates that the format of spatial representations is readily accessible to empirical investigation. 


\section{Transparency}

Action Editor: Marc J. Buehner

Editor: Patricia J. Bauer

Author Contributions

S. R. Yousif designed the experiments, collected the data, and analyzed the data with input from F. C. Keil. S. R. Yousif wrote the manuscript, and F. C. Keil provided critical revisions. Both authors approved the final manuscript for submission.

Declaration of Conflicting Interests

The author(s) declared that there were no conflicts of interest with respect to the authorship or the publication of this article.

Funding

This project was supported by a National Science Foundation Graduate Research Fellowship awarded to S. R. Yousif.

\section{Open Practices}

All data and experiment code have been made publicly available via OSF and can be accessed at https://osf.io/ tnhez. The design and analysis plans for Experiments 1a to $1 \mathrm{c}$ and Experiments $2 \mathrm{a}$ to $2 \mathrm{c}$ were preregistered on AsPredicted; copies of the preregistrations can be accessed at https://osf.io/tnhez. A minor deviation from the preregistration is discussed in the Method section for Experiment $2 \mathrm{a}$. This article has received the badges for Open Data, Open Materials, and Preregistration. More information about the Open Practices badges can be found at http:// www.psychologicalscience.org/publications/badges.

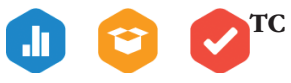

\section{ORCID iDs}

Sami R. Yousif (iD https://orcid.org/0000-0002-4126-3072 Frank C. Keil (ID https://orcid.org/0000-0003-3688-7558

\section{Acknowledgments}

For helpful conversation or comments on previous drafts, we thank Stella Lourenco, Jonathan Flombaum, Cameron Ellis, Brynn Sherman, the members of the Emory Spatial Cognition Lab, and the members of the Yale Cognition and Development Lab.

\section{Note}

1. Our claim that observers may use polar coordinates is not meant to suggest that they represent all locations on a single, inflexible polar grid. We mean to suggest only that, quite the opposite but similar to the cognitive-graph view, observers spontaneously use angle-distance relations to localize objects relative to other objects.

\section{References}

Algom, D., \& Fitousi, D. (2016). Half a century of research on Garner interference and the separability-integrality distinction. Psychological Bulletin, 142, 1352-1383.
Appelle, S. (1972). Perception and discrimination as a function of stimulus orientation: The "oblique effect" in man and animals. Psychological Bulletin, 78, 266-278.

Bays, P. M., Wu, E. Y., \& Husain, M. (2011). Storage and binding of object features in visual working memory. Neuropsychologia, 49, 1622-1631.

Coren, S., \& Girgus, J. S. (1978). Seeing is deceiving: The psychology of visual illusions. Erlbaum.

Dehaene, S., Bossini, S., \& Giraux, P. (1993). The mental representation of parity and number magnitude. Journal of Experimental Psychology: General, 122, 371-396.

Ericson, J. D., \& Warren, W. H. (2020). Probing the invariant structure of spatial knowledge: Support for the cognitive graph hypothesis. Cognition, 200, Article 104276. https:// doi.org/10.1016/j.cognition.2020.104276

Farah, M. J., Brunn, J. L., Wong, A. B., Wallace, M. A., \& Carpenter, P. A. (1990). Frames of reference for allocating attention to space: Evidence from the neglect syndrome. Neuropsychologia, 28, 335-347.

Firestone, C., \& Keil, F. C. (2016). Seeing the tipping point: Balance perception and visual shape. Journal of Experimental Psychology: General, 145, 872-881.

Gallistel, C. R. (1990). The organization of learning. MIT Press.

Garner, W. R., \& Felfoldy, G. L. (1970). Integrality of stimulus dimensions in various types of information processing. Cognitive Psychology, 1, 225-241.

Hubbard, T. L. (Ed.). (2018). Spatial biases in perception and cognition. Cambridge University Press.

Huttenlocher, J., Hedges, L. V., \& Duncan, S. (1991). Categories and particulars: Prototype effects in estimating spatial location. Psychological Review, 98, 352-376.

Kuipers, B., Tecuci, D. G., \& Stankiewicz, B. J. (2003). The skeleton in the cognitive map: A computational and empirical exploration. Environment and Behavior, 35, 81-106.

Langlois, T., Jacoby, N., Suchow, J. W., \& Griffiths, T. L. (2017). Uncovering visual priors in spatial memory using serial reproduction. In G. Gunzelmann, A. Howes, T. Tenbrink, \& E. J. Davelaar (Eds.), Proceedings of the 39th Annual Conference of the Cognitive Science Society (pp. 712-717). Cognitive Science Society.

Li, B., Peterson, M. R., \& Freeman, R. D. (2003). Oblique effect: A neural basis in the visual cortex. Journal of Neurophysiology, 90, 204-217.

McCloskey, M., \& Palmer, E. (1996). Visual representation of object location: Insights from localization impairments. Current Directions in Psychological Science, 5, 25-28.

McCloskey, M., Rapp, B., Yantis, S., Rubin, G., Bacon, W. F., Dagnelie, G., Gordon, B., Aliminosa, D., Boatman, D. F., Badecker, W., Johnson, D. N., Tusa, R. J., \& Palmer, E. (1995). A developmental deficit in localizing objects from vision. Psychological Science, 6, 112-117.

Moser, E. I., Kropff, E., \& Moser, M. B. (2008). Place cells, grid cells, and the brain's spatial representation system. Annual Reviews Neuroscience, 31, 69-89.

Moser, E. I., Roudi, Y., Witter, M. P., Kentros, C., Bonhoeffer, T., \& Moser, M. B. (2014). Grid cells and cortical representation. Nature Reviews Neuroscience, 15, 466-481. 
Müller, M., \& Wehner, R. (1988). Path integration in desert ants, Cataglyphis fortis. Proceedings of the National Academy of Sciences, USA, 85, 5287-5290.

O'Keefe, J., \& Nadel, L. (1978). The hippocampus as a cognitive map. Clarendon Press.

Olson, D. R. (2013). Cognitive development: The child's acquisition of diagonality. Psychology Press.

Palmer, S. E., Schloss, K. B., \& Sammartino, J. (2013). Visual aesthetics and human preference. Annual Review of Psychology, 64, 77-107.

Parkinson, C., \& Wheatley, T. (2013). Old cortex, new contexts: Re-purposing spatial perception for social cognition. Frontiers in Human Neuroscience, 7, Article 645. https://doi.org/10.3389/fnhum.2013.00645

Peirce, J., Gray, J. R., Simpson, S., MacAskill, M., Höchenberger, R., Sogo, H., Kastman, E., \& Lindeløv, J. K. (2019). PsychoPy2: Experiments in behavior made easy. Behavior Research Methods, 51, 195-203.

Pertzov, Y., \& Husain, M. (2014). The privileged role of location in visual working memory. Attention, Perception, $\varepsilon$ Psychophysics, 76, 1914-1924.
Warren, W. H., Rothman, D. B., Schnapp, B. H., \& Ericson, J. D. (2017). Wormholes in virtual space: From cognitive maps to cognitive graphs. Cognition, 166, 152-163.

Wedell, D. H., Fitting, S., \& Allen, G. L. (2007). Shape effects on memory for location. Psychonomic Bulletin \& Review, 14, 681-686.

Yang, F., \& Flombaum, J. (2018). Polar coordinates as the format of spatial representation in visual perception. Journal of Vision, 18(10), Article 21. https://doi.org/10.1167/18.10.21

Yousif, S. R., Chen, Y. C., \& Scholl, B. J. (2020). Systematic angular biases in the representation of visual space. Attention, Perception \& Psychophysics, 82, 3124-3143.

Yousif, S. R., \& Keil, F. C. (2019). The additive-area heuristic: An efficient but illusory means of visual area approximation. Psychological Science, 30, 495-503.

Yousif, S. R., \& Lourenco, S. F. (2017). Are all geometric cues created equal? Children's use of distance and length for reorientation. Cognitive Development, 43, 159-169.

Zorzi, M., Priftis, K., \& Umiltà, C. (2002). Brain damage: Neglect disrupts the mental number line. Nature, 417 , 138-139. 